\title{
On the Adjoint of a Strongly Continuous Semigroup
}

\author{
Diómedes Bárcenas ${ }^{1}$ and Luis Gerardo Mármol $^{2}$ \\ ${ }^{1}$ Universidad de los Andes, Mérida, Venezuela \\ ${ }^{2}$ Universidad Simón Bolivar, Caracas, Venezuela \\ Correspondence should be addressed to Luis Gerardo Mármol, lgmarmol@usb.ve
}

Received 1 December 2006; Revised 14 November 2007; Accepted 9 December 2007

Recommended by Simeon Reich

Using some techniques from vector integration, we prove the weak measurability of the adjoint of strongly continuous semigroups which factor through Banach spaces without isomorphic copy of $l_{1}$; we also prove the strong continuity away from zero of the adjoint if the semigroup factors through Grothendieck spaces. These results are used, in particular, to characterize the space of strong continuity of $\left\{T^{* *}(t)\right\}_{t \geq 0}$, which, in addition, is also characterized for abstract $L$ - and $M$-spaces. As a corollary, it is proven that abstract $L$-spaces with no copy of $l_{1}$ are finite-dimensional.

Copyright (C) 2008 D. Bárcenas and L. G. Mármol. This is an open access article distributed under the Creative Commons Attribution License, which permits unrestricted use, distribution, and reproduction in any medium, provided the original work is properly cited.

\section{Introduction}

The study of the adjoint of a strongly continuous semigroups was initiated by Phillips [1], who proved, among other things, that for the general case, the domain of continuity of the adjoint semigroup on a Banach space $X$ is different from $X^{*}$. A systematic study of the adjoint semigroup $\left\{T^{*}(t)\right\}_{t \geq 0}$ can be found in [2], where, among other things, the basic properties of $\left\{T^{*}(t)\right\}_{t \geq 0}$ and of the canonical spaces $X^{\odot}$ and $X^{\odot \odot}$ associated with it are studied. Also, in [2], Neerven introduces the space $X_{\odot \odot}$ and studies the relationship between

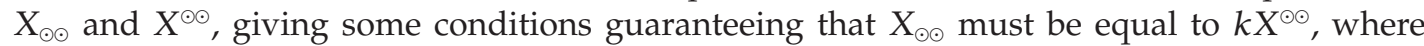
$k: X^{\odot \odot} \rightarrow X^{* *}$ is a natural embedding of $X^{\odot \odot}$ into $X^{* *}$, defined by the formula $\left\langle k x^{\odot \odot,}, x^{*}\right\rangle:=$ $\lim _{\lambda \rightarrow \infty}\left\langle x^{\odot \odot}, \lambda R\left(\lambda, A^{*}\right) x^{* *}\right\rangle, x^{\odot \odot} \in X^{\odot \odot}$.

Some of the principal tools used by van Neerven come from vector measures, like Gelfand and Pettis integrals, in order to provide the reader with some examples where the above-mentioned equality holds.

In this paper, we also employ certain techniques from vector measures to show that semigroups factoring through Banach spaces without isomorphic copy of $l_{1}$ have weakly measurable adjoint. On the other hand, we prove the strong continuity away from zero of the adjoint when the semigroup factors through Grothendieck spaces. These results have several 
applications; in particular, there is one related to our other main topic of interest: to find a characterization of the space $X_{\odot \odot}$ of strong continuity of a given second dual semigroup $\left\{T^{* *}(t)\right\}_{t \geq 0}$. This can be done in the case of spaces satisfying the above-mentioned hypotheses. This is also done when $X$ is sun-reflexive and $X^{\odot}$ is isomorphic to a dual Banach space. Additionally, we give a characterization of the strong continuity of $\left\{T^{* *}(t)\right\}_{t \geq 0}$ for abstract $L$ - and $M$-spaces, and prove that abstract $L$-spaces with no copy of $l_{1}$ are finite-dimensional.

\section{Preliminaries}

\subsection{The semigroup dual}

Let $X$ be a complex Banach space and let $\{T(t)\}_{t \geq 0}$ be a $C_{0}$-semigroup of bounded linear operators on $X$. The adjoint semigroup $\left\{T^{*}(t)\right\}_{t \geq 0}$ fails in general to be strongly continuous. The semigroup dual of $X$ with respect to $\{T(t)\}_{t \geq 0}$, notation $X^{\odot}$ (pronounced $X$-sun) is defined as the linear subspace of $X^{*}$ on which $\left\{T^{*}(t)\right\}_{t \geq 0}$ acts in a strongly continuous way

$$
X^{\odot}=\left\{x^{*} \in X^{*}: \lim _{t \downarrow 0}\left\|T^{*}(t) x^{*}-x^{*}\right\|=0\right\} .
$$

From this definition, we have that $X^{\odot}$ is $T^{*}(t)$-invariant, that is, $T^{*} X^{\odot} \subseteq X^{\odot}$, for all $t \geq 0$.

It can also be shown (see, e.g., [2]) that $X^{\odot}$ is a closed, weak ${ }^{*}$-dense linear subspace of $X^{*}$.

\subsection{The space $X^{\odot \odot}$}

Let $\left\{T^{\odot}(t)\right\}_{t \geq 0}$ denote the restriction of $\left\{T^{*}(t)\right\}_{t \geq 0}$ to the $T^{*}(t)$-invariant subspace $X^{\odot}$. Since $X^{\odot}$ is closed, $X^{\odot}$ is a Banach space. Also, it is clear from the definition of $X^{\odot}$ that $\left\{T^{\odot}(t)\right\}_{t \geq 0}$ is a strongly continuous semigroup on $X^{\odot}$.

We can also define $\left\{T^{\odot *}(t)\right\}_{t \geq 0}$ to be the adjoint of $\left\{T^{\odot}(t)\right\}_{t \geq 0}$ and write $X^{\odot \odot}$ for $\left(X^{\odot}\right)^{\odot}$ (read as $X$-sun-sun or $X$-bosom).

If $j: X \rightarrow X^{\odot *}$ is defined by $\left\langle j x, x^{\odot}\right\rangle:=\left\langle x^{\odot}, x\right\rangle$, then $\|j\| \leq 1$ and $j(X) \subseteq X^{\odot \odot}$. Moreover, $j$ is an embedding and we can identify $X$ isomorphically with the closed subspace $j X$ of $X^{\odot \odot}$. If $j X=X^{\odot \odot}$, then $X$ is said to be sun-reflexive or $\odot$-reflexive with respect to $\{T(t)\}_{t \geq 0}$.

The following results can be found in [2].

Theorem 2.1. Let $X$ be a complex Banach space, $\{T(t)\}_{t \geq 0}$ a $C_{0}$-semigroup on $X$ with infinitesimal generator $A$, satisfying $\|T(t)\| \leq M e^{w t}$, and let $A^{*}$ be the adjoint of $A$.

The formula

$$
\left\langle k x^{\odot \odot}, x^{*}\right\rangle:=\lim _{\lambda \rightarrow \infty}\left\langle x^{\odot \odot}, \lambda R\left(\lambda, A^{*}\right) x^{*}\right\rangle, \quad x^{\odot \odot} \in X^{\odot \odot},
$$

defines a natural embedding $k: X^{\odot \odot} \rightarrow X^{* *}$. Moreover, $1 \leq\|k\| \leq M$ and $\left.k x^{\odot \odot}\right|_{X^{\odot}}=x^{\odot \odot}$. If $i: X \rightarrow X^{* *}$ is the natural map, then we have $i=k j$.

We also have the following characterization of $k X^{\odot \odot}$.

Theorem 2.2. It holds that

$$
k X^{\odot \odot}=\left\{x^{* *} \in X^{* *}: \lim _{\lambda \rightarrow \infty} \lambda R(\lambda, A)^{* *} x^{* *}=x^{* *}\right\} .
$$


The former theorem suggests to define the following.

Definition 2.3.

$$
X_{\odot \odot}:=\left\{x^{* *} \in X^{* *}: \lim _{t \downarrow 0} T^{* *}(t) x^{* *}=x^{* *}\right\} .
$$

A natural question is: do the spaces $X_{\odot \odot}$ and $k X^{\odot \odot}$ coincide? Trivially, this is true if $X^{*}=X^{\odot}$, since then we have the coincidence of both definitions. It is also shown in [2] that $k$ maps $X^{\odot \odot}$ into $X_{\odot \odot}$, and so $k X^{\odot \odot} \subseteq X_{\odot \odot}$ always holds, but this inclusion can be proper. More specifically,

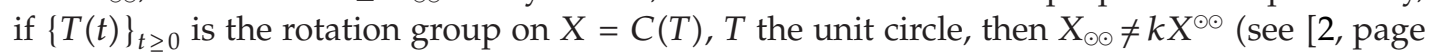
99]). to $k X^{\odot \odot}$.

In the following, we will be interested in giving conditions guaranteeing that $X_{\odot \odot}$ is equal

\subsection{The Gelfand and Pettis integrals}

Let $(\Omega, \Sigma, \mu)$ be finite measure space and let $X$ be a Banach space. Suppose that $f: \Omega \rightarrow X^{*}$ is weakly ${ }^{*}-\mu$-measurable and suppose further that for each $x \in X$ the function $\langle f(\cdot), x\rangle$ belongs to $L^{1}(\mu)$. For each $E \in \Sigma$ define a map $T_{E}: X \rightarrow L^{1}(\mu), T_{E} x=\left\langle f X_{E}(\cdot), x\right\rangle$. It can be easily shown that $T_{E}$ is bounded. This implies that the linear map $x_{E}^{*}$ defined by

$$
\left\langle x_{E}^{*}, x\right\rangle:=\int_{E}\langle f(\cdot), x\rangle d \mu
$$

is bounded. The element $x_{E}^{*} \in X^{*}$ is called the weak*-integral or Gelfand integral of $f$ over $E$ with respect to $\mu$, notation $x_{E}^{*}=$ weak $^{*} \int_{E} f d \mu$.

We have from the definition that the weak ${ }^{*}$-integral satisfies

$$
\left\langle\text { weak }^{*} \int_{E} f d \mu, x\right\rangle=\int_{E}\langle f(\cdot), x\rangle d \mu
$$

for all $E \in \Sigma$ and $x \in X$.

Now, if $f: \Omega \rightarrow X$ is weakly $\mu$-measurable and for each $x^{*} \in X^{*}$, the function $\left\langle x^{*}, f(\cdot)\right\rangle$ belongs to $L^{1}(\mu)$, then using the same argument as above, each $E \in \Sigma$ defines an element $x^{* *} \in X^{* *}$ such that

$$
\left\langle x_{E}^{* *}, x^{*}\right\rangle=\int_{E}\left\langle x^{*}, f(\cdot)\right\rangle d \mu
$$

for all $x^{*} \in X^{*}$. If for all $E \in \Sigma$ the element $x_{E}^{* *}$ belongs to $X$, then $f$ is said to be Pettis integrable with respect to $\mu$.

For a detailed study of the Gelfand and Pettis integrals, see [3-5].

Theorem 2.4. An element $x_{\odot \odot} \in X_{\odot \odot}$ belongs to $k X^{\odot \odot}$ if and only if for all $t>0$ and $x^{*} \in X^{*}$ one has

$$
\left\langle x_{\odot \odot}, w e a k^{*} \int_{0}^{t} T^{*}(\sigma) x^{*} d \sigma\right\rangle=\int_{0}^{t}\left\langle x_{\odot \odot}, T^{*}(\sigma) x^{*}\right\rangle d \sigma .
$$

For a proof of this, see [2]. 
Corollary 2.5. $X_{\odot \odot}=k X^{\odot \odot}$ if and only if for all $t>0, x^{*} \in X^{*}$, and $x_{\odot \odot} \in X_{\odot \odot}$ one has

$$
\left\langle x_{\odot \odot,}, w e a k^{*} \int_{0}^{t} T^{*}(\sigma) x^{*} d \sigma\right\rangle=\int_{0}^{t}\left\langle x_{\odot \odot}, T^{*}(\sigma) x^{*}\right\rangle d \sigma .
$$

Hence, if $\left\{T^{*}(t)\right\}_{t \geq 0}$ is Pettis integrable, that is, for all $x^{*} \in X^{*}$ and $\tau>0$, the map $t \rightarrow$ $T^{*}(t) x^{*}$ is Pettis integrable on $[0, \tau]$, then $X_{\odot \odot}=k X^{\odot \odot}$. In particular, this holds if $\left\{T^{*}(t)\right\}_{t \geq 0}$ is strongly continuous away from zero (notation: $C_{>0}$ ).

Definition 2.6. Let $f, g: \Omega \rightarrow X$ be two weakly $\mu$-measurable functions. One says that $f, g$ are weakly $\mu$-equivalent if $\left\langle x^{*}, f(\cdot)\right\rangle=\left\langle x^{*}, g(\cdot)\right\rangle \mu$-almost everywhere, for each $x^{*} \in X^{*}$. If $f, g: \Omega \rightarrow$ $X^{*}$ are weakly ${ }^{*} \mu$-measurable, one says that $f, g$ are weakly ${ }^{*} \mu$-equivalent if $\langle f(\cdot), x\rangle=\langle g(\cdot), x\rangle$ for each $x \in X$.

Following [5], we will denote by $\mathcal{D}(\mu, X)$ the space of classes of weakly $\mu$-equivalent Pettis $\mu$-integrable $X$-valued functions. It is a linear space with ordinary algebraic operations.

Definition 2.7. $X$ has the $\mu$-weak Radon-Nikodym property ( $\mu$-WRNP) if for each $X$-valued $\mu$ continuous measure of $\sigma$-finite variation $\gamma$, there exists $f \in D(\mu, X)$ such that

$$
\left\langle x^{*}, \gamma(E)\right\rangle=\int_{E}\left\langle x^{*}, f\right\rangle d \mu
$$

for each $x^{*} \in X^{*}$ and $E \in \Sigma$.

$X$ has the WRNP if it has the $\mu$-WRNP for every $\mu$.

For more details about the WRNP, see $[4,5]$.

One of the principal tools used here is the following theorem.

Theorem 2.8. $X^{*}$ has the weak Radon-Nikodym property if and only if X contains no isomorphic copy of $l^{1}$.

\section{Strong continuity of the adjoint semigroup}

It is well known that a semigroup is strongly continuous if and only if it is weakly continuous (see [6]). In [7], Bárcenas and Diestel have proved that if a $C_{0}$-semigroup $\{T(t)\}_{t \geq 0}$ is such that, for each $t>0, T(t)$ factors through an Asplund space, then the adjoint semigroup $\left\{T^{*}(t)\right\}_{t \geq 0}$ is $C_{>0}$. (A Banach space $X$ is an Asplund space if and only if $X^{*}$ has the Radon-Nikodym property.) Since weakly compact operators factor through reflexive Banach spaces (which are Asplund spaces), we see that the adjoint semigroup of a semigroup of weakly compact operators is strongly continuous away from $O$ (see [8]). Bárcenas and Diestel used those results to get some applications in optimal control theory.

In this section, we get the same conclusion of Bárcenas-Diestel if a $C_{0}$-semigroup $\{T(t)\}_{t \geq 0}$ is such that, for each $t>0, T(t)$ factors through a Grothendieck space.

We recall that a Banach space is a Grothendieck space if every weakly ${ }^{*}$-convergent sequence in $X^{*}$ is also weakly convergent. Equivalently, $X$ is a Grothendieck space if every linear bounded operator from $X$ to any separable space, Banach space is weakly compact. Among Grothendieck spaces, we list all reflexive Banach spaces and $L^{\infty}(\Omega, \Sigma, \mu)$, where $(\Omega, \Sigma, \mu)$ is 
a positive measure space. A Banach space isomorphic to a complemented subspace of a Grothendieck space is also a Grothendieck space. Several characterizations of Grothendieck spaces are found in [9].

A Banach space is said to have the Dunford-Pettis property if every weakly compact operator in $L(X)$ applies relatively weakly compact sets onto relatively norm compact sets. The most common examples of Banach spaces with the Dunford-Pettis property are $L^{1}(\mu)$ and $C(K)$. Complemented subspaces of a space with the Dunford-Pettis property have also the Dunford-Pettis property. For more details about this, see [10].

If $X$ is a Grothendieck space with the Dunford-Pettis property, Lotz (see [11]) has shown that every strongly continuous semigroup is uniformly continuous and so the adjoint semigroup is uniformly continuous.

We also recall that a bounded linear operator $T: X \rightarrow Y$ (where $X$ and $Y$ are Banach spaces) factors through a Banach space $Z$ if there are bounded linear operators $u: X \rightarrow Z$ and $v: Z \rightarrow Y$ such that the following diagram:

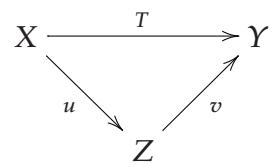

commutes.

Theorem 3.1. Let $X$ be a Banach space and $\{T(t)\}_{t \geq 0}$ a $C_{0}$-semigroup defined on $X$. Suppose that for every $a>0$ there exists a Grothendieck space $Y_{a}$ such that $T(a)$ factors through $Y_{a}$. Then $\left\{T^{*}(t)\right\}_{t \geq 0}$ is $C_{>0}$.

Proof. More generally, we will prove that, given $a>0$, the adjoint semigroup is strongly continuous for $t \geq a$ if the operator $T(a)$ factors through a Grothendieck space $Y_{a}$.

Let $a$ be a positive number. There exist a Grothendieck space $Y_{a}$ and bounded linear operators $u: X \rightarrow Y_{a}$ and $v: Y_{a} \rightarrow X$ such that the following diagram is commutative:

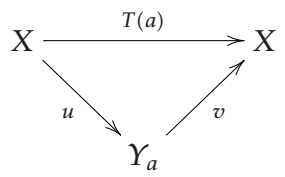

For every $t>a$, the following diagram also commutes, due to semigroup properties:

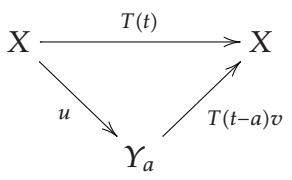

Hence $v^{*} T^{*}(t-a)=(T(t-a) v)^{*}$ is $w^{*}$-continuous. If $\left(t_{n}\right) \in(a,+\infty)$ and $t_{n} \rightarrow t$, then $v^{*} T^{*}\left(t_{n}-a\right) \stackrel{w^{*}}{\rightarrow} v^{*} T^{*}(t-a)$. But $Y_{a}$ is a Grothendieck space, and consequently $v^{*} T^{*}\left(t_{n}-a\right) \stackrel{w}{\rightarrow}$ $v^{*} T^{*}(t-a)$. From this, we can deduce that $v^{*} T^{*}(t-a)$ is weakly continuous for $t \geq a$. Finally, $u^{*}(T(t-a) v)^{*}=(T(t-a) v u)^{*}=T(t)^{*}$ is $w$-continuous for $t \geq a$, which implies that $\left\{T^{*}(t)\right\}_{t \geq a}$ is strongly continuous. 
Remark 3.2. Our proof that semigroups factorizing through Grothendieck spaces have adjoint semigroup $C_{>0}$ can be adapted to prove that the adjoint of a $C_{0}$-semigroup on a Grothendieck space is also a $C_{0}$-semigroup. This result can also be obtained by combining the results of 2.3.2 and 2.3.3 from [12].

Remark 3.3. Since reflexive Banach spaces are Grothendieck spaces, this gives an alternative proof that $C_{0}$-semigroups of weakly compact operators have adjoint semigroups which are $C_{>0}$, thanks to the Davis, Figiel, Johnson, and Pelczynski factorization scheme (see [8]).

Remark 3.4. Theorem 3.1 also has applications in optimal control theory. See [7] for details.

The following theorem shows, in the spirit of Remark 3.3, new examples of semigroups satisfying the hypotheses of Theorem 3.1.

Theorem 3.5. Let $\{T(t)\}_{t \geq 0}$ be a strongly continuous semigroup on an AL-space. If, for $t>0, T(t) X$ does not contain any isomorphic copy of $l^{1}$, then $\{T(t)\}_{t \geq 0}$ is a compact semigroup.

Proof. Let $X$ be an $A L$-space and $T(t): X \rightarrow X$ a strongly continuous semigroup such that for $t>0, T(t) X$ does not contain any isomorphic copy of $l^{1}$. Then $T(t)$ applies bounded sets onto sets which contain a weakly Cauchy sequence. Since $A L$-spaces are weakly sequentially complete, $T(t)$ applies bounded sets onto weakly compact sets, due to the Eberlein-Smulian theorem. Therefore $T(t)$ is weakly compact. Since $A L$-spaces have the Dunford-Pettis property, the square of a weakly compact operator is compact; hence for $t>0$ we have

$$
T(t)=T\left(\frac{t}{2}\right)^{2}
$$

with $T(t / 2)$ being weakly compact, $T(t)$ is compact.

Corollary 3.6. Every infinite-dimensional AL-space contains a copy of $l^{1}$.

Proof. If not, we consider the semigroup defined, for each $t$ by $T(t):=I$, the identity on $X$, which is strongly continuous and hence compact, and, by the Riesz lemma, $X$ is finite-dimensional.

We finish this section with two results related to the aim of finding a characterization of the space $X_{\odot \odot}$ of strong continuity of $\left\{T^{* *}(t)\right\}_{t \geq 0}$.

Theorem 3.7. If $X$ is $\odot$-reflexive under $\{T(t)\}_{t \geq 0}$ and $X^{\odot}$ is isomorphic to a dual Banach space, then $\left\{T^{*}(t)\right\}_{t \geq 0}$ is $C_{>0}$.

Proof. $X$ is $\odot$-reflexive if and only if $X^{\odot}$ is $\odot$-reflexive (see [2]). If $X$ is $\odot$-reflexive, then the resolvent $R(\lambda, A)$ is weakly compact. Since

$$
X=\overline{D(A)}=\overline{\text { linspan } R(\lambda, A) B_{X}}
$$

we can conclude that each $\odot$-reflexive Banach space is necessarily weakly compactly generated.

Then $X^{\odot}$ is weakly compactly generated and therefore $X^{\odot}$ has the Radon-Nikodym property since weakly compactly generated dual Banach spaces have that property (see [13]). Now $[2$, Corollary 6.2.4] is applied. 
Theorem 3.8. If $X$ is an AM-space or an $A L$-space, and $\{T(t)\}_{t \geq 0}$ is a $C_{0}$-semigroup on $X$, then $\left\{T^{* *}(t)\right\}_{t \geq 0}$ is strongly continuous if and only if $\{T(t)\}_{t \geq 0}$ has bounded generator.

Proof. If $X$ is an $A M$-space, then $X^{*}$ is an $A L$-space and therefore there exists $(\Omega, \Sigma, \mu)$ such that $X$ is isometrically isomorphic to $L^{1}(\mu)$ (see [14, page 18] for details). So $X^{* *}$ is isometrically isomorphic to $L^{\infty}(\mu)$, which is a Grothendieck space with the Dunford-Pettis property. Since $\left\{T^{* *}(t)\right\}_{t \geq 0}$ is a $C_{0}$-semigroup, it has bounded generator, by the Lotz theorem. So $\{T(t)\}_{t \geq 0}$ has bounded generator.

Now we suppose that $X$ is an $A L$-space and $\left\{T^{* *}(t)\right\}_{t \geq 0}$ is strongly continuous. By [12, Lemma II.3.2], $\left\{T^{*}(t)\right\}_{t \geq 0}$ is strongly continuous. Since $X^{*}$ is a Grothendieck space with the Dunford-Pettis property, it has bounded generator. Therefore $\{T(t)\}_{t \geq 0}$ has bounded generator.

\subsection{The weak measurability of the adjoint semigroup}

Definition 3.9. Let $K$ be a compact Hausdorff space. A function $\phi: K \rightarrow \mathbb{C}$ is said to be universally measurable if it is $\mu$-measurable for all finite positive regular Borel measures $\mu$ on $K$. A function $\psi: K \rightarrow X$, with $X$ a Banach space, is universally weakly measurable if $\left\langle x^{*}, \psi(\cdot)\right\rangle$ is universally measurable for all $x^{*} \in X^{*} . \psi$ is called universally Pettis integrable if it is Pettis integrable with respect to every $\mu$.

The following theorem of Riddle-Saab and Uhl (see [15]) is useful in providing examples of $C_{0}$-semigroups for which $X_{\odot \odot}=k X^{\odot \odot}$.

Theorem 3.10. Let $X$ be a separable Banach space and suppose that $\psi: K \rightarrow X^{*}$ is a bounded, universally weakly measurable function. Then $\psi$ is universally Pettis integrable.

Remark 3.11. If $\left\{T^{*}(t)\right\}_{t \geq 0}$ is weakly Borel measurable, that is, for all $x^{*} \in X^{*}$ and $\tau>0$ the map $t \rightarrow T^{*}(t) x^{*}$ is weakly Borel measurable on $[0, \tau]$, it follows from Theorem 3.10 that $\left\{T^{*}(t)\right\}_{t \geq 0}$ is Pettis integrable. Combining this with Corollary 2.5 and the notes immediately following it, we get.

Corollary 3.12. Suppose that $X$ is separable. If $\left\{T^{*}(t)\right\}_{t \geq 0}$ is weakly Borel measurable, then $X_{\odot \odot}=$ $k X^{\odot \odot}$.

Remark 3.13. It should be pointed out that weak measurability does not imply strong measurability. For example, let JF be the James function space, defined as the completion of the linear span of the characteristic functions of subintervals of $[0,1]$ with respect to the norm

$$
\|f\|=\sup \left(\sum_{i=0}^{k-1}\left|\int_{t_{i}}^{t_{i+1}} f(t) d t\right|^{2}\right)^{1 / 2},
$$

where the supremum runs over all partitions $0=t_{0}<t_{1}<\cdots<t_{k}=1$ of $[0,1]$. Define $T(t)$ on JF by

$$
T(t) f=f(x+t \bmod 1) .
$$

It is shown [2, pages 159-160] that the adjoint semigroup $\left\{T^{*}(t)\right\}_{t \geq 0}$ is weakly Borel measurable (and hence Pettis integrable) but not $C_{>0}$. In particular, it cannot be strongly measurable. 
As it has been noted by van Neerven, as a consequence of the theorem of Odell-Rosenthal (see [16]), if $X$ is separable and does not contain a closed subspace isomorphic to $l^{1}$, then each $x^{* *} \in X^{* *}$ is the weak ${ }^{*}$-limit of some sequence in $X$.

If $\{T(t)\}_{t \geq 0}$ is a $C_{0}$-semigroup on such a space, then $\left\langle x^{* *}, T^{*}(t) x^{*}\right\rangle$ is the pointwise limit of the continuous functions $\left\langle T^{*}(t) x^{*}, x_{n}\right\rangle$, which implies that it is Borel measurable. From this, we can deduce that $X_{\odot \odot}=k X^{\odot \odot}$.

Now, using the WRNP of the dual space of any space with no copy of $l^{1}$, we will prove that if $T(t)$ factors through a separable space without copy of $l^{1}$, then $\left\{T^{*}(t)\right\}_{t \geq 0}$ is weakly measurable, thus giving some generality to the former results.

We will begin with a weak ${ }^{*}$ version of the theorem of Riddle-Saab and Uhl.

Theorem 3.14. Let $X$ be a Banach space with no copy of $l^{1}$ and suppose that $\psi: K \rightarrow X^{*}$ is a bounded, universally weakly measurable function. Then $\psi$ is weak ${ }^{*}$-equivalent to a Pettis integrable function.

Proof. Let $\gamma_{\psi}: \Sigma \rightarrow X^{*}$ be the weak ${ }^{*}$-integral of $\psi$, that is,

$$
\left\langle x, \gamma_{\psi}(E)\right\rangle=\int_{E}\langle x, \psi\rangle d \mu
$$

for each $x \in X$ and $E \in \Sigma$. $\gamma_{\psi}$ is weakly*-countably additive. $X$ does not contain a subspace isomorphic to $l^{1}$. Then, by a classic result of Bessaga and Pelczynski (see [17, pages 48-49]), $X^{*}$ does not contain a copy of $l_{\infty}$. By [5, Theorem 4.2, pages 197-198] (see also [3, I.4.7]), $\gamma_{\psi}$ is a measure in the strong topology of $X^{*}$.

Now we use the WRNP of $X^{*}$ : there exists an $\mu$-Pettis integrable function $g$ such that

$$
\left\langle x, \gamma_{\psi}(E)\right\rangle=\int_{E}\langle x, g\rangle d \mu .
$$

By (3.8) and (3.9), we conclude that $\langle x, \psi\rangle=\langle x, g\rangle \mu$-almost everywhere, for all $x \in X$.

Theorem 3.15. Let $X$ be a Banach space and let $\{T(t)\}_{t \geq 0}$ be a $C_{0}$-semigroup defined on $X$. If for every $t>0, T(t)$ factors through a separable Banach space with no copy of $l^{1}$, then $\left\{T^{*}(t)\right\}_{t \geq 0}$ is weakly Borel measurable.

Proof. Let $[a, b]$ be any closed and bounded interval contained in $(0, \infty)$. There exists a Banach space $Y_{a}$ with no copy of $l^{1}$, and bounded linear operators $u: X \rightarrow Y_{a}$ and $v: Y_{a} \rightarrow X$ such that the following diagram is commutative:

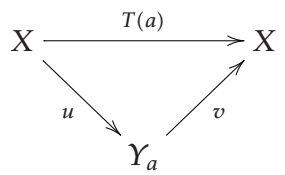

For every $t \in[a, b]$, the following diagram also commutes, due to semigroup property:

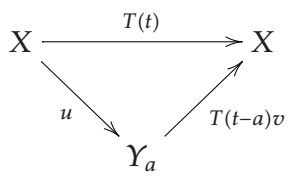


Then $v^{*} T^{*}(t-a)$ is weak ${ }^{*}$-continuous and hence Gelfand integrable with respect to the Lebesgue measure on $[a, b]$.

For each $E \subseteq[a, b]$, we define

$$
\gamma(E)=\int_{E} v^{*} T^{*}(t-a) x^{*} d t
$$

where $\gamma(E)$ is a countably additive vector measure which is also a vector measure in the strong topology, by the same argument used in the proof of Theorem 3.14. On the other hand, we have that $\gamma$ is absolutely continuous with respect to the Lebesgue measure on $[a, b]$.

By the WRNP of $Y_{a}^{*}$, there exists a Pettis-integrable function $f$ such that

$$
r(E)=\int_{E} f(t) d t
$$

Let $\left\{y_{n}\right\}_{n \in \mathbb{N}}$ be a dense subset in $Y_{a}$. From (3.12) and (3.13), we have

$$
y_{n} \gamma(E)=\int_{E} y_{n} f(t) d t=\int_{E} y_{n} v^{*} T^{*}(t-a) x^{*} d t
$$

for each $n \in \mathbb{N}$.

Let $\mu$ denote the Lebesgue measure on $[a, b]$. According to (3.14), there exists $N_{n}$, $\mu\left(N_{n}\right)=0$ such that

$$
y_{n} f(t)=y_{n} v^{*} T^{*}(t-a) x^{*}
$$

for every $t \in[a, b] \backslash N_{n}$.

Now, take $N=\bigcup_{n=1}^{\infty} N_{n}$; then $\mu(N)=0$ and

$$
y_{n} f(t)=y_{n} v^{*} T^{*}(t-a) x^{*}
$$

for every $t \in[a, b] \backslash N_{n}$ and $n \in \mathbb{N}$.

Since $\left\{y_{n}\right\}_{n \in \mathbb{N}}$ is dense in $Y_{a}$, we have that

$$
f(t)=v^{*} T^{*}(t-a) x^{*}
$$

for every $t \in[a, b] \backslash N$, and hence $v^{*} T_{t-a}^{*}$ is weakly Borel measurable. Finally

$$
u^{*}(T(t-a) v)^{*}=(T(t-a) v u)^{*}=(T(t-a) T(a))^{*}=T^{*}(t)
$$

is also weakly Borel measurable.

Theorem 3.16. Let $X$ and $\{T(t)\}_{t \geq 0}$ be as in Theorem 3.15. Then $X_{\odot \odot}=k X^{\odot \odot}$.

Proof. We are going to prove that $\left\{T^{*}(t)\right\}_{t \geq 0}$ is Pettis integrable.

As in Theorem 3.15 , Let $[a, b] \subseteq(0, \infty)$ by any closed bounded interval. We have the following factorization scheme:

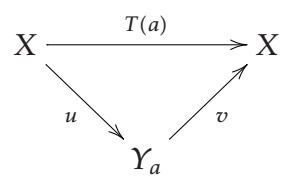


we also have

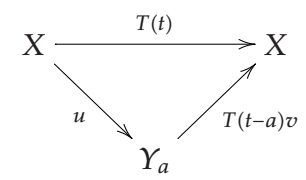

Then, using the same argument, we conclude that there exists a Pettis-integrable function $f$ : $[a, b] \rightarrow X^{*}$ such that

$$
v^{*} T^{*}(t-a) x^{*}=f(t) \text { almost everywhere }
$$

but then

$$
T^{*}(t) x^{*}=u^{*}(f(t))
$$

and it is easy to see that $u^{*}(f(t))$ is Pettis integrable.

Remark 3.17. A Banach space $X$ is said to have the Lotz property if every strongly continuous semigroup on $X$ has bounded generator. It is noticeable that Leung [18] has found a Lotz space without the Dunford-Pettis property, and it is also noticeable that to be a Grothendieck space is not enough to ensure the Lotz property, since in $l^{p}, 1<p<\infty$, we can easily define a $C_{0^{-}}$ semigroup which is not uniformly continuous by means of

$$
\begin{gathered}
T(t): l^{p} \longrightarrow l^{p}, \\
x:=\sum_{n=1}^{\infty} \alpha_{n} x_{n} \longmapsto T(t) x:=\sum_{n=1}^{\infty} \alpha_{n} e^{-n t} x_{n},
\end{gathered}
$$

where $\left\{x_{n}\right\}$ is the standard unit basis.

Reworking on van Neerven results, we can show the relationship that exists among some of them (note that they were stated separately), thus giving necessary and sufficient conditions to ensure the uniform continuity of the adjoint of a strongly continuous semigroup defined on a Banach space whose dual has the Lotz property. This can be done as follows.

Let $X$ be a complex Banach space such that $X^{*}$ has the Lotz property, and let $\{T(t)\}_{t \geq 0}$ be a $C_{0}$-semigroup on $X$ with infinitesimal generator $A$.

The following statements are equivalent.

(1) $\{T(t)\}_{t \geq 0}$ is uniformly continuous.

(2) $\left\{T^{*}(t)\right\}_{t \geq 0}$ is strongly continuous.

(3) $\lim _{\lambda \rightarrow \infty}\left\|\lambda R\left(\lambda, A^{*}\right) x^{*}-x^{*}\right\|=0$ for all $x^{*} \in X^{*}$.

(4) $\lim _{t \downarrow 0} \|(1 / t)\left(\right.$ weak $\left.^{*} \int_{o}^{t} T^{*}(\sigma) x^{*} d \sigma\right)-x^{*} \|=0$ for all $x^{*} \in X^{*}$, where weak $\int_{o}^{t} T^{*}(\sigma) x^{*} d \sigma$ is the Gelfand integral of $T^{*}(\sigma)$ on $[0, t]$ with respect to the Lebesgue measure.

(5) The quotient semigroup on $X^{*} / X^{\odot}$ is strongly continuous.

\section{Acknowledgment}

This work is partially supported by CDCH of ULA under Project C-1335-05-05-A and by Universidad Simón Bolivar, Caracas, Venezuela. 


\section{References}

[1] R. S. Phillips, "The adjoint semigroup," Pacific Journal of Mathematics, vol. 5, pp. 269-283, 1955.

[2] J. van Neerven, The Adjoint of a Semigroup of Linear Operators, vol. 1529 of Lecture Notes in Mathematics, Springer, Berlin, Germany, 1992.

[3] J. Diestel and J. J. Uhl Jr., Vector Measures, Mathematical Surveys, no. 15, American Mathematical Society, Providence, RI, USA, 1977.

[4] K. Musiał, "Pettis integral," in Handbook of Measure Theory, Vol. I, II, E. Pap, Ed., chapter 12, pp. 531-586, North-Holland, Amsterdam, The Netherlands, 2002.

[5] K. Musiał, "Topics in the theory of Pettis integration," Rendiconti dell'Istituto di Matematica dell'Università di Trieste, vol. 23, no. 1, pp. 177-262, 1991.

[6] K. Yosida, Functional Analysis, vol. 123 of Die Grundlehren der mathematischen Wissenschaften, Springer, New York, NY, USA, 4th edition, 1974.

[7] D. Bàrcenas and J. Diestel, "Constrained controllability in nonreflexive Banach spaces," Quaestiones Mathematicae, vol. 18, no. 1-3, pp. 185-198, 1995.

[8] W. J. Davis, T. Figiel, W. B. Johnson, and A. Pełczyński, "Factoring weakly compact operators," Journal of Functional Analysis, vol. 17, pp. 311-327, 1974.

[9] J. Diestel, "Grothendieck spaces and vector measures," in (Proc. Sympos., Alta, Utah, 1972), Vector and Operator Valued Measures and Applications, pp. 97-108, Academic Press, New York, NY, USA, 1973.

[10] J. Diestel, A Survey of Results Related to the Dunford-Pettis Property, vol. 2 of Contemporary Mathematics, American Mathematical Society, Providence, RI, USA, 1980.

[11] H. P. Lotz, "Uniform convergence of operators on $L^{\infty}$ and similar spaces," Mathematische Zeitschrift, vol. 190, no. 2, pp. 207-220, 1985.

[12] R. Nagel, Ed., One-Parameter Semigroups of Positive Operators, vol. 1184 of Lecture Notes in Mathematics, Springer, Berlin, Germany, 1986.

[13] T. H. Kuo, "On conjugate Banach spaces with the Radon-Nikodym property," Pacific Journal of Mathematics, vol. 59, no. 2, pp. 497-503, 1975.

[14] J. Lindenstrauss and L. Tzafriri, Classical Banach Spaces. II. Sequence Spaces, Springer, Berlin, Germany, 1977.

[15] L. H. Riddle, E. Saab, and J. J. Uhl Jr., "Sets with the weak Radon-Nikodym property in dual Banach spaces," Indiana University Mathematics Journal, vol. 32, no. 4, pp. 527-541, 1983.

[16] E. Odell and H. P. Rosenthal, "A double-dual characterization of separable Banach spaces containing $l^{1}, "$ Israel Journal of Mathematics, vol. 20, no. 3-4, pp. 375-384, 1975.

[17] J. Diestel, Sequences and Series in Banach Spaces, vol. 92 of Graduate Texts in Mathematics, Springer, New York, NY, USA, 1984.

[18] D. Leung, "Uniform convergence of operators and Grothendieck spaces with the Dunford-Pettis property," Mathematische Zeitschrift, vol. 197, no. 1, pp. 21-32, 1988. 


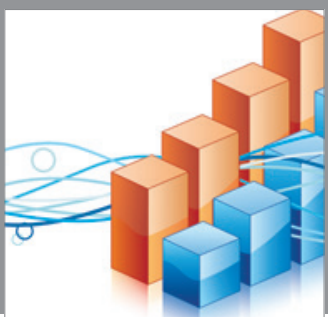

Advances in

Operations Research

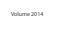

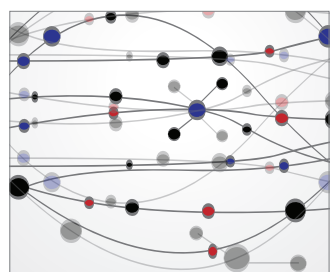

\section{The Scientific} World Journal
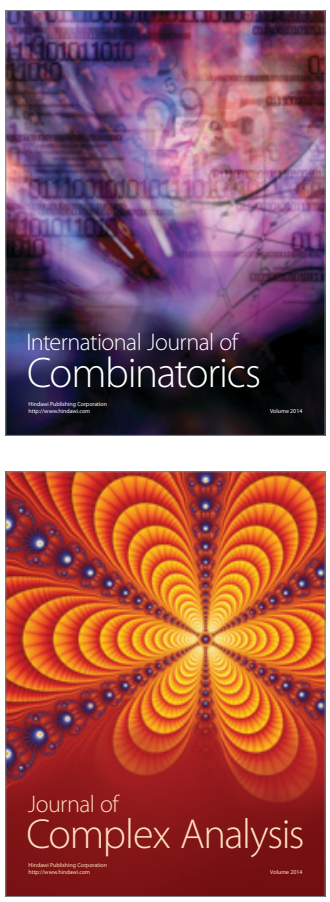

International Journal of

Mathematics and

Mathematical

Sciences
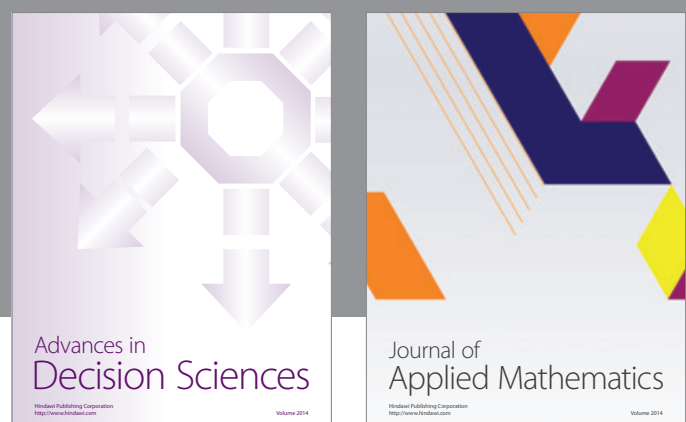

Journal of

Applied Mathematics
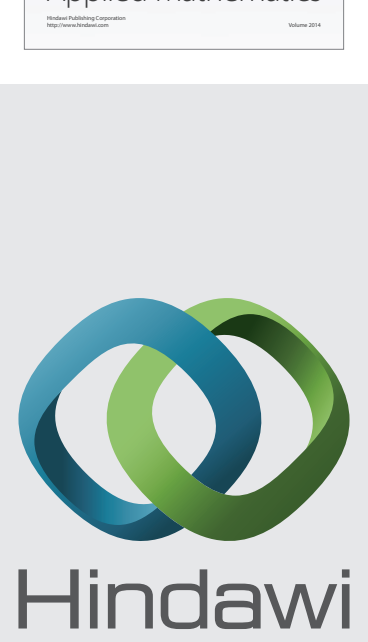

Submit your manuscripts at http://www.hindawi.com
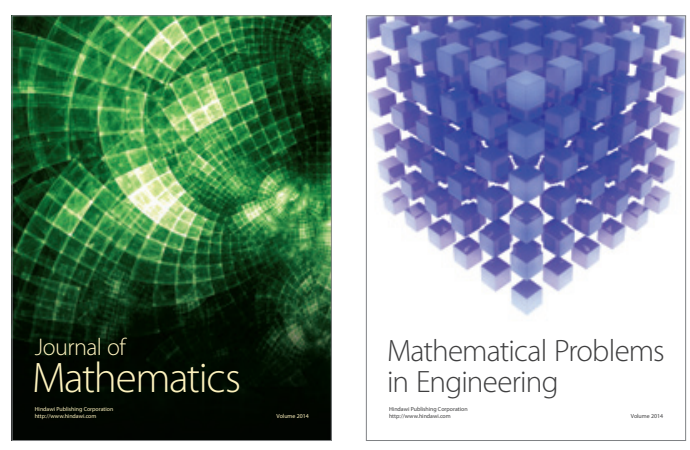

Mathematical Problems in Engineering
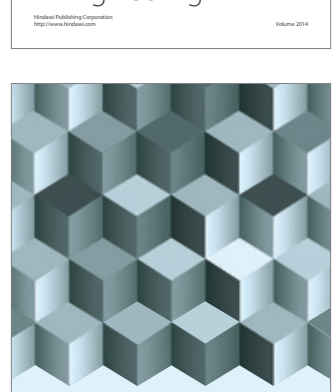

Journal of

Function Spaces
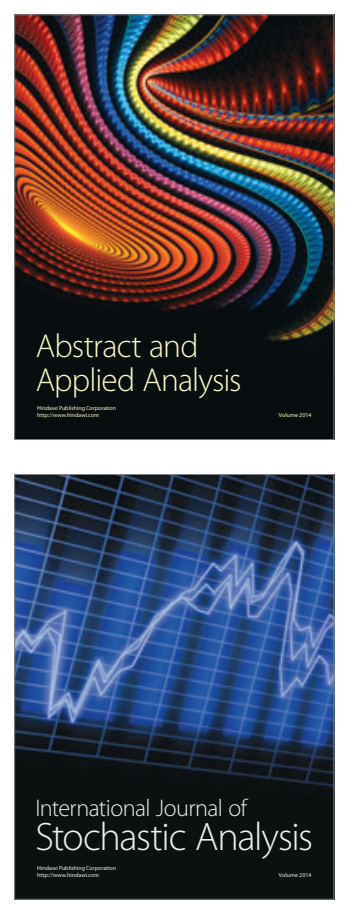

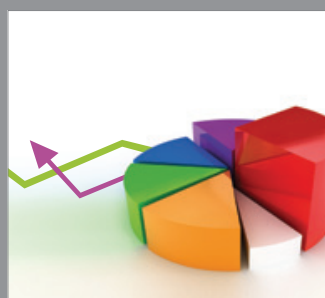

ournal of

Probability and Statistics

Promensencen
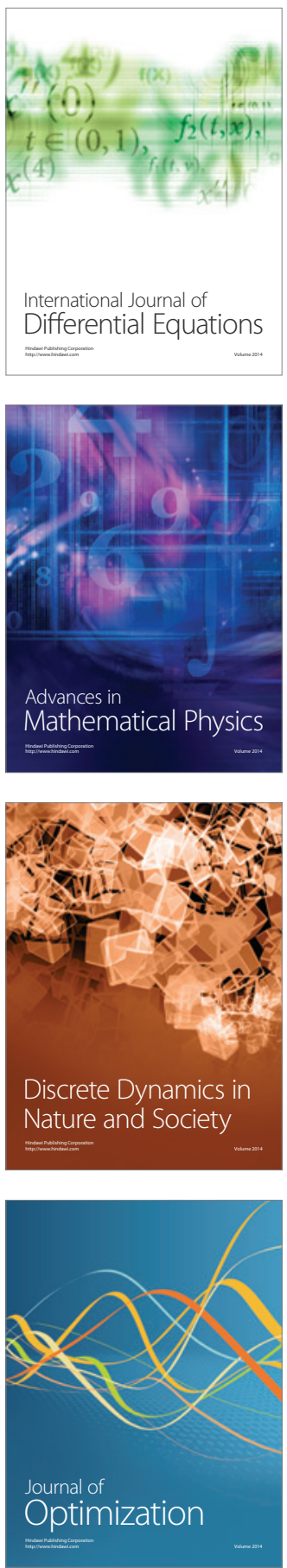